\title{
Editorial
}

\section{Human-Centric Intelligent Systems: A Welcome Note from Editor(s)-in-Chief}

The Human-Centric Intelligent Systems journal is dedicated to publishing latest research concerning theoretical and practical aspects of the healthy, responsible, and positive relation between intelligent technology and humans. Human-centric intelligence is becoming significant because various digital systems surrounding humans are increasingly upgrading because of human input while presenting an effective experience between humans and digital systems. By developing machine intelligence and systems with a goal of understanding human language, emotion and behavior, and interactions between humans and technological systems, human-centric intelligent systems push the boundaries of previously limited artificial intelligence (AI) solutions to bridge the gap between machine and human.

In past decades, the trajectory of human daily activities among human being and interacted with cyber space has led to a large amount of human-centric digital information in an unprecedented scale, depth and breadth, which presents urgent challenges of studying effective methodologies, techniques and technical tools for capturing, representing, modeling, analyzing, understanding and managing human behaviors, social and cultural phenomenon and dynamics both in physical and cyber world. Multiple categories of information sources, digital behavior trajectories within communities, and advancements in behavioral and social computing have the potential to address these challenges such as information diffusion, community dynamics, trust, privacy, and security issues, and open-up a new multidisciplinary research field of human-centric intelligence to better serve the humanity, make the technology more humane and rightful.

The importance of Human-Centric Intelligent Systems is not to replace humans entirely but to enhance our capabilities by way of intelligence. Although the current advanced techniques such as machine learning, deep learning, and AI are being widely explored for enhancing the scalability, reliability, responsibility, and capability, new extensions are required that will allow researchers and developers to conceive and design novel applications to take advantage of the new paradigms and technologies. However, these challenges are prompting researchers to create knowledge that requires new avenues of publishing, sharing, and dissemination. Therefore, we proudly launch a new journal, Human-Centric Intelligent Systems, to the scientific community and would like to warmly welcome our readers, contributors, scientific editors and editorial board members of the Human-Centric Intelligent Systems journal. At insights into Human-Centric Intelligent Systems, we aim to solicit the latest research findings and practical experiences of various theories and practical applications in human-centric intelligent systems and provide theoretical and algorithmic insights in human-centric computing and analytics frontier.

Human-Centric Intelligent Systems, encourages topics related but are not limited to the followings topics, which cover the current key research interest in managing the interactions between users, organizations, communities, and computing systems, and modeling and governance of social and organizational concepts, e.g., community, trust, credibility, and privacy:

- Human-Centric Data and Management

- Information Diffusion and Modeling

- Diffusion Source Identification and Network Analysis

- Classification, Ranking, Summarization, and Recommendation

- Social Influence Analysis

- Community Detection and Dynamics

- Disinformation and Misinformation

- Network Structure and Community Evolution Analysis

- User Modeling, Personalization, and Recommendation

- Responsible AI, Fairness, and Explainability

- Behavioral/Choice Modeling

- Behavioral Dynamics and Simulation

- User Behavior and Influence Analysis

- Community/Crowd Behavioral Analysis

- Event Tracking and Detection

- Trust Computing and Privacy Preserving

- Social and Ethical Issue Analysis

- Intelligent System Design and Evaluation

- Mobile, Ubiquitous, and Pervasive Sensing

- Data Mining in Mobile and Social Sensing

- Robust Visual Encoding Methods 
- Robust Visual Interactive System Design and Evaluation for Data Visualization

- Healthcare, Mobility, Economics and Society, etc.

These are the hot topics that will satisfy the ever-changing needs of a world-wide audience. With the development of computing and AI technology, and interdisciplinary movement between science, technology, and social science, new emerging topics will be added to the journal scope, to reflect future trends of human-centric intelligent systems.

We welcome four types of submissions reporting latest research findings of all theoretical and practical applications in human-centric intelligent systems, and cutting-edge theoretical and algorithmic insights in human-centric computing and analytics, which include

- Research Article. A research article describes an original study which is of interest to the readership of the journal.

- Review Article. A review article is a comprehensive and balanced survey of recent developments in a given field which is of interest to the readership of the journal.

- Perspective. A perspective is intended to provide a forwardlooking and/or speculative approach to a given research topic which is of interest to the readership of the journal. Perspectives should always remain balanced and aim to stimulate discussion within the relevant research community.

- Correspondence. A correspondence is a concise communication which provides a forum to address new or hot issues which are relevant to the readership of the journal.

Tianrui $\mathrm{Li}^{1}$, Guandong $\mathrm{Xu}^{2, *}$

${ }^{1}$ School of Computing and Artificial Intelligence, Southwest Jiaotong University, Chengdu, China

${ }^{2}$ School of Computer Science, University of Technology Sydney, Sydney, Australia

"Corresponding author. Email: Guandong.Xu@uts.edu.au

Peer review under responsibility of KEO (Henan) Education Technology Co. Ltd
We are pleased to state that Human-Centric Intelligent Systems has gathered a very prestigious editorial board members hailing from around the world from backgrounds of Data Science, AI, Machine Learning, Information Systems, Behavior Science, and Applied Psychology, who form a spectrum of complementary knowledge covering relevant topics. We are thankful to all editorial board members for their willingness and commitment to serve the editorial mission of Human-Centric Intelligent Systems. The list of members who have joined us in this endeavor is available at https://www.atlantis-press.com/journals/hcis/editorial-board. In addition, we are honored to have an advisory board, which comprises of highly respected researchers and academicians from around the world. They are:

- Masaru Kitsuregawa, University of Tokyo, Tokyo, Japan

- Philip S. Yu, University of Illinois at Chicago, Chicago, United States

Human-Centric Intelligent Systems is an open access journal, i.e. all articles are immediately and permanently free to read, download, copy \& distribute. The journal is published under the CC BY-NC 4.0 user license which defines the permitted third-party reuse of its articles. Publication in this journal is free of charge for Authors. All open access publication fees are subsidized by KEO (Henan) Education Technology Co. Ltd (KEO). Note that this is a promotional offer which applies to all papers submitted before December 31, 2022.

With Best Regards from Managing Editors, the whole Editorial Board and Editor(s)-in-Chief. 